\title{
Damping of Linear Nonadiabatic MHD Waves in a Flowing Prominence Medium
}

\author{
Nagendra Kumar, ${ }^{1}$ Anil Kumar, ${ }^{2}$ Himanshu Sikka, ${ }^{2}$ and Pradeep Kumar ${ }^{3}$ \\ ${ }^{1}$ Department of Mathematics, M.M.H. College, Ghaziabad 201001, Uttar Pradesh, India \\ ${ }^{2}$ Department of Mathematics, Maharaja Agrasen Institute of Technology, Rohini 110086, Delhi, India \\ ${ }^{3}$ Department of Physics, K.G.K (P.G.) College, Moradabad 244001, Uttar Pradesh, India
}

Correspondence should be addressed to Anil Kumar; anilk_kang@yahoo.com

Received 23 December 2013; Revised 25 March 2014; Accepted 19 April 2014; Published 15 May 2014

Academic Editor: Wing Huen Ip

Copyright (C) 2014 Nagendra Kumar et al. This is an open access article distributed under the Creative Commons Attribution License, which permits unrestricted use, distribution, and reproduction in any medium, provided the original work is properly cited.

\begin{abstract}
We study the effect of shear flow on the time damping of linear nonadiabatic magnetoacoustic waves in a solar prominence. We consider a homogeneous, isothermal, and unbounded medium permeated by a uniform magnetic field. The adiabaticity is removed by including the optically thin radiative losses, thermal conduction, and heating term in energy equation. We present a local theory of MHD waves to obtain a dispersion relation. The dispersion relation is solved numerically to study the time damping of these waves. It is found that flow influences the damping time and damping per period of both the slow and fast waves significantly. Damping time and damping per period of slow waves are very much higher than the damping time and damping per period of fast waves.
\end{abstract}

\section{Introduction}

A large number of observations of oscillations in large scale magnetic structures such as prominence have been observed from space-borne and ground based instruments [1-5]. Small amplitude oscillations observed in quiescent solar prominence can be interpreted in terms of linear magnetohydyrodynamic waves. To study these small amplitude oscillations, one of the damping mechanism nonadiabaticities has been extensively studied because linear nonadiabatic magnetoacoustic waves have interesting physical effects such as time and spatial damping of the disturbances. Carbonell et al. [6] studied the time damping of linear nonadiabatic magnetohydrodynamic waves in an unbounded plasma with coronal properties and found that behavior of the damping changes significantly when going from very small to very large wavenumbers and becomes almost constant for very large wavenumbers. Spatial damping of linear nonadiabatic magnetoacoustic waves in prominence medium has been studied and it is found that the spatial damping is dominated by thermal conduction at shorter periods while the spatial damping is dominated by radiation at the periods greater than $1 \mathrm{~s}$ [7]. Soler et al. [8] showed that nonadiabatic effects are efficient damping mechanism for magnetoacoustic modes.

Ground and space-borne satellites observations have confirmed that the solar and space plasmas are always dynamic showing flow [9-11]. Therefore, all the theoretical models should include the presence of flow. Nakariakov and Roberts [12] theoretically investigated the effect of steady mass flow in coronal and photospheric slabs. Carbonell et al. [13] discussed the combined effects of nonadiabatic mechanism and steady flow on the time damping of slow modes and found that flow does not modify the damping time but increases the period of slow mode dramatically for the flow velocities very close to nonadiabatic sound speed. In all previous studies, shear flow has not been taken into account. Therefore, in the present 
study, we investigate the local behavior of time damping of linear nonadiabtic magnetoacoustic waves in the presence of shear flow.

\section{Basic Equations and Dispersion Relation}

The equilibrium configuration considered in the present work is made of a homogeneous slab that is infinite in all directions. The magnetic field is uniform and is oriented along the $z$-direction. The background flow in $z$-direction is assumed to vary linearly in the $x$-direction as $\mathbf{v}_{0}=v_{0}(x) \widehat{z}=$ $a x \widehat{z}$, with a constant $a$ [14]. This assumption of the linear profile for velocity shear is applicable for wide variety of astrophysical shear flows. Parallel thermal conduction to the magnetic field, heating, and radiative losses are considered as nonadiabatic effects. The gravitational effects have been neglected because wavelengths are assumed much smaller than gravitational scale height. The basic MHD equations describing the plasma motion are given by

$$
\begin{gathered}
\frac{D \rho}{D t}+\rho \nabla \cdot \mathbf{v}=0 \\
\rho\left[\frac{\partial}{\partial t}+\mathbf{v} \cdot \nabla\right] \mathbf{v}+\nabla p=\frac{(\nabla \times \mathbf{B}) \times \mathbf{B}}{4 \pi}, \\
\frac{\partial \mathbf{B}}{\partial t}=\nabla \times(\mathbf{v} \times \mathbf{B}), \\
\frac{1}{\gamma-1} \frac{\partial p}{\partial t}-\frac{\gamma p}{\rho(\gamma-1)} \frac{\partial \rho}{\partial t}+\rho L(\rho, T)-\nabla \cdot(\kappa \cdot \nabla T)=0, \\
p=\frac{2 \rho k_{\mathbf{B}} T}{m_{p}} .
\end{gathered}
$$

Here, $\rho, p, T, \mathbf{v}$, and $\mathbf{B}$ denote the density, gas pressure, temperature, velocity, and magnetic field, respectively. The term $\nabla \cdot(\kappa \cdot \nabla T)$ represents the thermal conduction. The symbol $L$ is heat-loss function and is given by

$$
L(\rho, T)=\chi \rho T^{\alpha},
$$

where $\chi$ and $\alpha$ are piecewise continuous functions depending on temperature [6].

Considering small perturbations from the equilibrium, the field quantities can be written as

$$
\begin{gathered}
\mathbf{B}=\mathbf{B}_{\mathbf{0}}+\mathbf{B}_{1}(\mathbf{r}, t), \quad \mathbf{v}=\mathbf{v}_{0}(\mathbf{x})+\mathbf{v}_{1}(\mathbf{r}, t), \\
p=p_{0}+p_{1}(\mathbf{r}, t), \quad \rho=\rho_{0}+\rho_{1}(\mathbf{r}, t), \\
T=T_{0}+T_{1}(\mathbf{r}, t)
\end{gathered}
$$

where the subscripts " 0 " and " 1 " refer to equilibrium and perturbed quantities, respectively. Here, the equilibrium magnetic field $\mathbf{B}_{\mathbf{0}}$ is homogeneous and is taken along $z$-axis; that is, $\mathbf{B}_{\mathbf{0}}=B_{0} \widehat{z}$. The assumption about a homogeneous magnetic field may be reasonable for the large regions of quite sun but does not imply appropriate results. However, the assumption of a homogeneous magnetic field provides us a relatively simple mathematical model to study MHD waves in solar prominence. Several authors $[6,15,16]$ used homogeneous magnetic field to study MHD waves in different regions of solar atmosphere. In the future, we will consider inhomogeneous magnetic field to study such MHD waves in solar prominence model and the results will be reported elsewhere.

We linearise the basic equations (1) to obtain

$$
\begin{gathered}
\frac{\partial \rho_{1}}{\partial t}+\left(\mathbf{v}_{0} \cdot \nabla\right) \rho_{1}+\rho_{0}\left(\nabla \cdot \mathbf{v}_{1}\right)+\rho_{1}\left(\nabla \cdot \mathbf{v}_{0}\right)=0 \\
\rho_{0} \frac{\partial \mathbf{v}_{1}}{\partial t}+\rho_{0} v_{0} \frac{\partial \mathbf{v}_{1}}{\partial z}+\rho_{0} v_{x} \frac{\partial \mathbf{v}_{0}}{\partial x}=-\nabla p_{1}-\frac{\mathbf{B}_{0} \times\left(\nabla \times \mathbf{B}_{1}\right)}{4 \pi} \\
\frac{\partial \mathbf{B}_{\mathbf{1}}}{\partial t}=\nabla \times\left(\mathbf{v}_{0} \times \mathbf{B}_{1}\right)+\nabla \times\left(\mathbf{v}_{1} \times \mathbf{B}_{\mathbf{0}}\right) \\
\frac{\partial p_{1}}{\partial t}-c_{s}^{2} \frac{\partial \rho_{1}}{\partial t}+(\gamma-1)\left[L_{\rho} \rho_{1}+L_{T} T_{1}\right] \rho_{0} \\
=(\gamma-1)\left(\mathbf{B}_{0} \cdot \nabla\right)\left[\frac{\kappa_{\|}}{B_{0}^{2}}\left(\mathbf{B}_{0} \cdot \nabla\right) T_{1}\right] \\
\frac{p_{1}}{p_{0}}=\frac{\rho_{1}}{\rho_{0}}+\frac{T_{1}}{T_{0}}
\end{gathered}
$$

where $\kappa_{\|}=10^{-11} T^{5 / 2} \mathrm{Wm}^{-1} \mathrm{~K}^{-1}$ is thermal conduction parallel to magnetic field. If background flow is spatially inhomogeneous in the vertical direction, we can not apply Fourier transformation and a nonlocal approach would be more appropriate in this context. Though the local approximation has limited applicability, in the present study we are interested in investigating the behavior of nonadiabatic waves in the initial stage of the evolution of wave perturbations. Since local approximation is restricted to short wavelength fluctuations, (4) can be analyzed using Fourier transform over space and time for the study of local behavior of waves [17-20]. So we perform Fourier analysis in plane waves and assume that all perturbations are of the form $e^{i(\mathbf{k} \cdot \mathbf{r}-\omega t)}$, where $\mathbf{k}=k_{x} \widehat{x}+$ $k_{z} \widehat{z}$ is the wavenumber and $\omega$ is the frequency. From linear equations (4), we obtain the following dispersion relation:

$$
\begin{aligned}
& \left(\Omega \omega\left(\Omega+i v_{0}^{\prime}\right)-k^{2} v_{A}^{2} \omega+i v_{A}^{2} k_{x} k_{z} v_{0}^{\prime}\right)\left(\Omega A_{1} \omega+\frac{k_{z}^{2}}{\rho_{0}} A_{2}\right) \\
& +\frac{k_{x}^{2} \Omega^{2} \omega}{\rho_{0}} A_{2}=0,
\end{aligned}
$$

where

$$
\begin{gathered}
\Omega=\omega-k_{z} v_{0}, \quad A_{1}=\left(k_{T} c_{s}-i \omega\right), \\
A_{2}=\frac{\rho_{0} c_{s}^{3}}{\gamma}\left(k_{\rho}-k_{T}\right)+i \rho_{0} \omega c_{s}^{2}, \\
k_{T}=\frac{A T_{0}}{p_{0} c_{s}}, \quad k_{\rho}=\frac{H \rho_{0}}{p_{0} c_{s}}, \\
A=(\gamma-1) \kappa_{\|} k_{x}^{2}+(\gamma-1) \rho_{0} L_{T}
\end{gathered}
$$




$$
\begin{gathered}
H=(\gamma-1)\left(L+\rho_{0} L_{\rho}\right), \\
L_{\rho}=\left(\frac{\partial L}{\partial \rho}\right)_{T}, \quad L_{T}=\left(\frac{\partial L}{\partial T}\right)_{\rho} .
\end{gathered}
$$

Here, $v_{A}$ is the Alfven velocity, $c_{s}$ is the sound velocity, and $v_{0}^{\prime}$ is the derivative of $v_{0}$ with respect to $x$. If we simplify the dispersion relation (5), we can obtain a sixth order polynomial in $\omega$ due to flow. Since we are solving this dispersion relation numerically, there is no need to simplify it in $\omega$.

In the absence of flow, the dispersion relation (5) reduces to

$$
\begin{gathered}
\omega\left(\omega^{5}+i k_{T} c_{s} \omega^{4}-k^{2}\left(c_{s}^{2}+v_{a}^{2}\right) \omega^{3}\right. \\
-i k^{2}\left(v_{a}^{2} k_{T} c_{s}+c_{s}^{3}\left(\frac{k_{T}-k_{\rho}}{\gamma}\right)\right) \omega^{2} \\
\left.+k^{2} v_{a}^{2} k_{z}^{2} c_{s}^{2} \omega-i k^{2} v_{a}^{2} k_{z}^{2} c_{s}^{3} \frac{\left(k_{\rho}-k_{T}\right)}{\gamma}\right)=0 .
\end{gathered}
$$

Equation (7) yields the following fifth order dispersion relation in $\omega$ :

$$
\begin{aligned}
\omega^{5} & +i k_{T} c_{s} \omega^{4}-k^{2}\left(c_{s}^{2}+v_{a}^{2}\right) \omega^{3} \\
& -i k^{2}\left(v_{a}^{2} k_{T} c_{s}+c_{s}^{3}\left(\frac{k_{T}-k_{\rho}}{\gamma}\right)\right) \omega^{2} \\
& +k^{2} v_{a}^{2} k_{z}^{2} c_{s}^{2} \omega-i k^{2} v_{a}^{2} k_{z}^{2} c_{s}^{3} \frac{\left(k_{\rho}-k_{T}\right)}{\gamma}=0
\end{aligned}
$$

which is similar to that obtained by Carbonell et al. [6] and it has been discussed by them in detail. So we will here solve and discuss the dispersion relation (5) to study the damping time and damping per period of fast and slow waves.

\section{Results and Discussion}

We solve the dispersion relation (5) numerically taking density, temperature, and magnetic field for the prominence region [21] corresponding to quiescent prominence conditions of solar atmosphere. The values of equilibrium parameters for the above mentioned prominence region are taken as $T_{0}=8000 \mathrm{~K}, \rho_{0}=5 \times 10^{-11} \mathrm{~kg} \mathrm{~m}^{-3}, B_{0}=5 \mathrm{G}, \alpha=7.4$, and $\chi=1.76 \times 10^{-13}$ (Hildner [21]). Here we consider the real wavenumber and find the complex solutions of the frequency as $\omega_{r}+i \omega_{i}$. We obtain two pairs of roots representing the fast and slow modes whereas purely imaginary root corresponds to thermal mode. The roots thus obtained do not represent instability because of small values of flow. For each pair $\left(\omega_{1}, \omega_{2}\right), \omega_{1}=\omega_{r}-i \omega_{i}$ and $\omega_{2}=-\omega_{r}-i \omega_{i}, \omega_{1}$

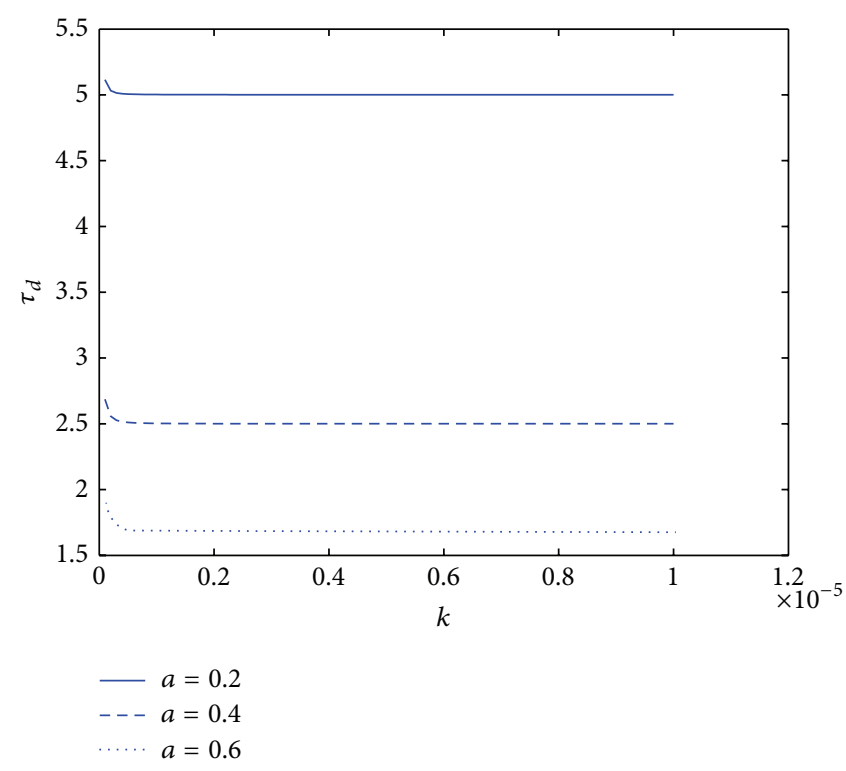

FIgURE 1: Damping time $\tau_{d}$ of fast waves versus wavenumber $k$.

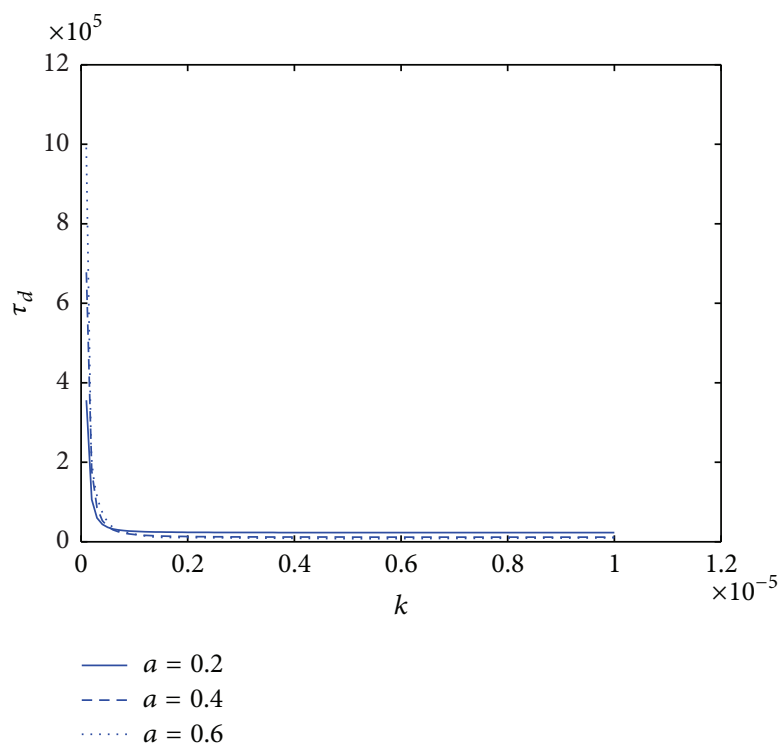

FIGURE 2: Damping time $\tau_{d}$ of slow waves versus wavenumber $k$.

corresponds to the parallel wave propagating towards the positive $z$-direction, whereas $\omega_{2}$ corresponds to antiparallel wave propagating towards negative $z$-direction. The damping time is $\tau_{d}=1 / \omega_{i}$ and damping per period is $D_{p}=\omega_{i} / \omega_{r}$. We plot Figures 1-6 to study the effect of flow on damping time and damping per period of these waves with wavenumber. Figure 1 shows the variation of damping time of fast wave with wavenumber whereas Figure 2 shows the variation of damping time of slow wave with wavenumber for different values of flow. Damping time of both slow and fast waves decreases with the increase in the value of flow. For smaller values of wavenumber $k$ damping time is higher than the larger values of wavenumber and becomes constant after 


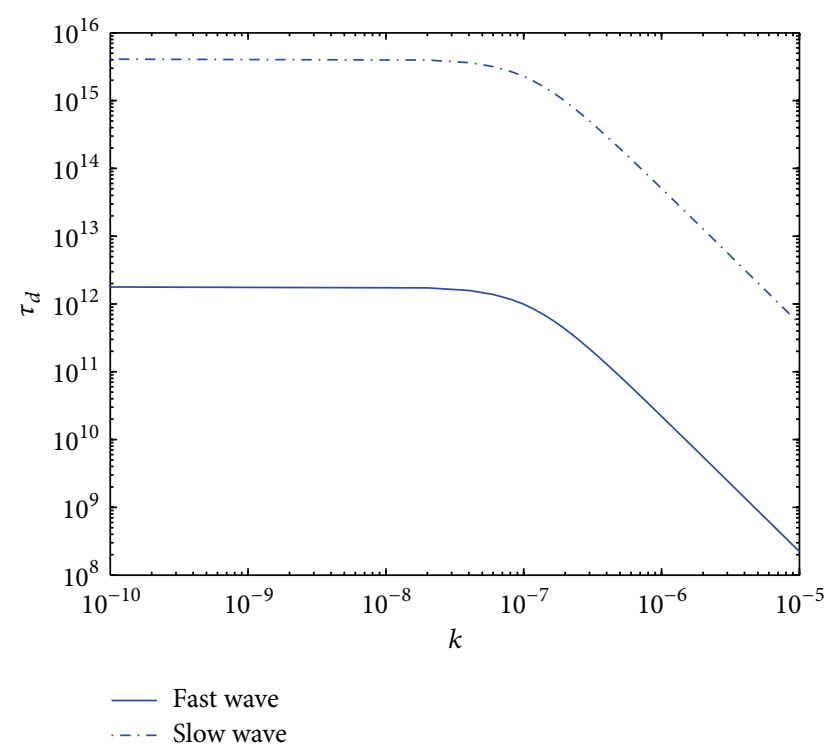

FIGURE 3: Damping time $\tau_{d}$ versus wavenumber $k$.

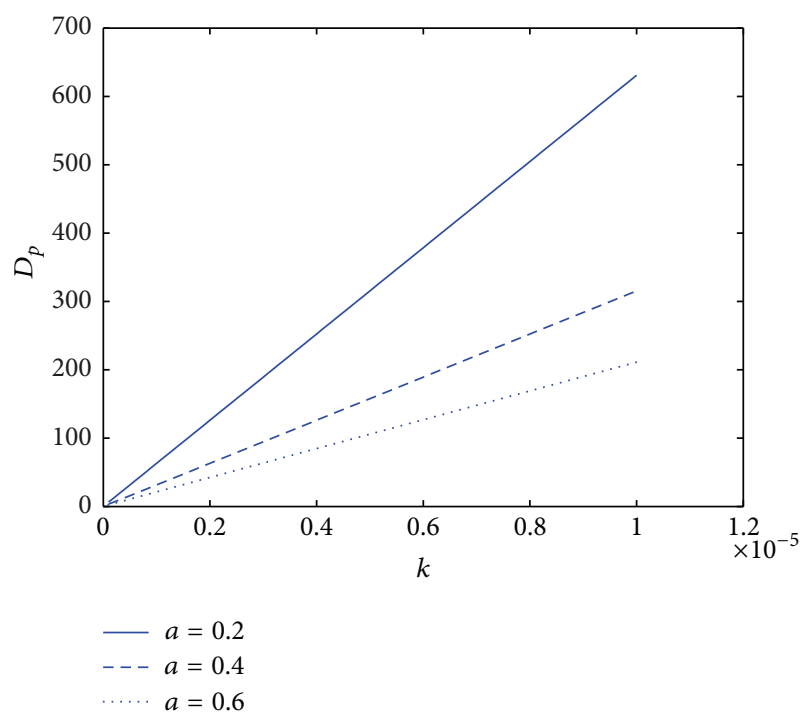

FIGURE 4: Damping per period $D_{p}$ of fast waves versus wavenumber $k$.

a certain value of wavenumber. Damping time of slow wave is very much higher than the fast wave corresponding to the same set of values of flow as considered in fast wave. In the absence of flow, Figure 3 has been drawn to see the variation of damping time for slow and fast wave by changing $\mathrm{X}$ - and Y-scales from linear to log according to N. Kumar and P. Kumar [22] and it is found that results shown in Figure 3 are in agreement with results shown in Figure 1(b) of [22].

Figures 4 and 5 are drawn for damping per period versus wavenumber for fast and slow waves for the same set of values of flow, respectively. Damping per period of slow waves is very much higher than the damping per period of fast waves corresponding to similar values of flow. The patterns

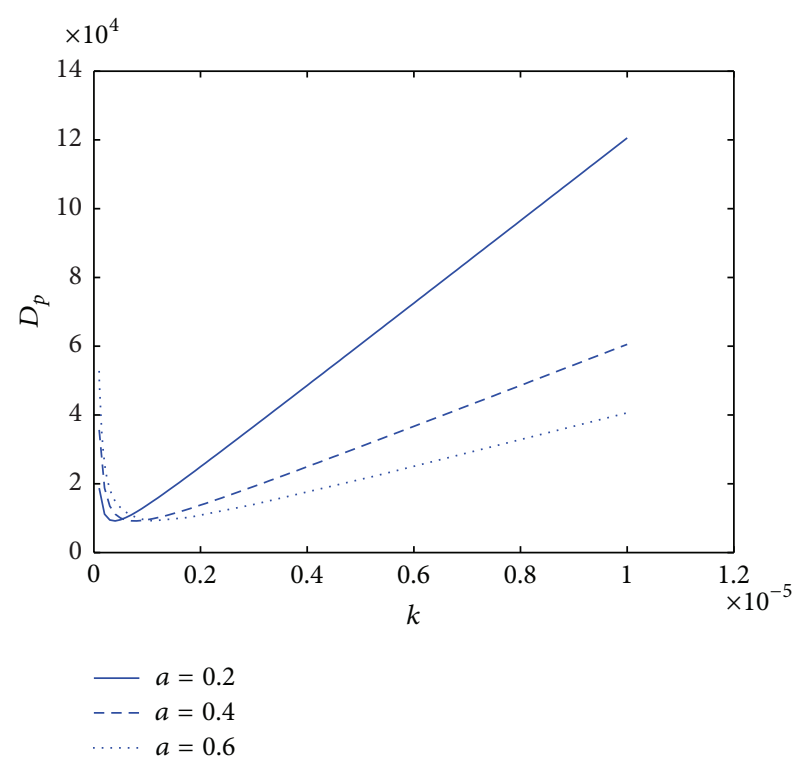

FIGURE 5: Damping per period $D_{p}$ of slow waves versus wavenumber $k$.

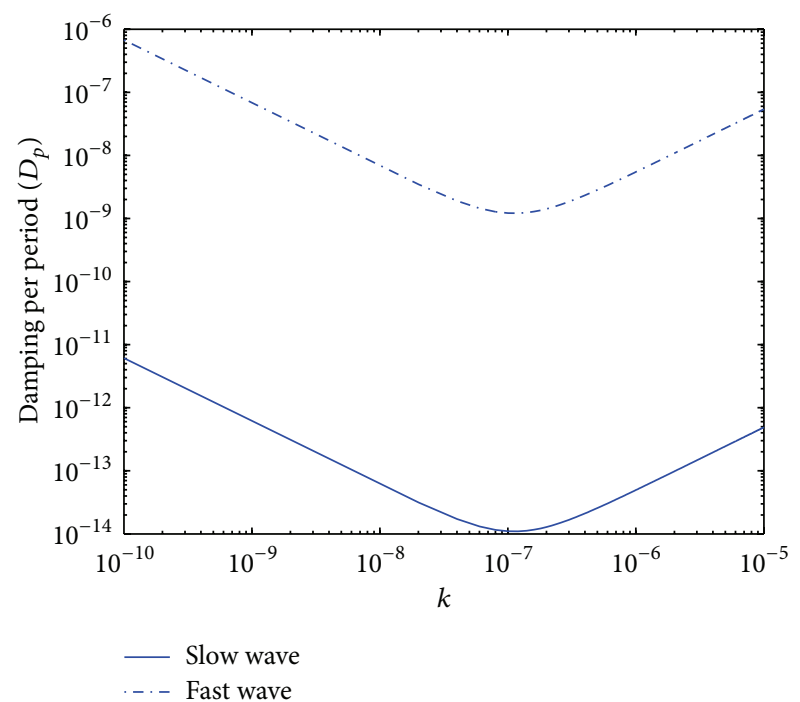

FIGURE 6: Damping per period $D_{p}$ versus wavenumber $k$.

of increasing damping per period are almost similar for both slow and fast waves. Damping per period for both waves has linear dependence on wavenumber except for smaller values of $k$ in case of slow waves. Figure 6 drawn in log scale depicts that the pattern of variation of damping per period is similar to that shown in Figure 1(c) by N. Kumar and P. Kumar [22] in the absence of flow.

\section{Conclusions}

Quiescent solar prominences and filaments are those features in solar atmosphere which show material flows and oscillations. So, in this paper, we have analyzed the time damping of 
MHD waves in nonadiabatic solar prominence plasma taking into account the effects of thermal conduction and shear flow. The nonadiabaticity is modeled by a general heat-loss function of thermodynamic parameters of plasma. We have obtained the dispersion relation using local approximation and solved it numerically to see the variations of damping time and damping per period of slow and fast MHD waves with wavenumber for different values of flow parameter. It is found that damping time of slow and fast waves decreases with the increase in flow and damping time of the slow wave is very much higher than that of the fast wave. It is observed that damping per period also decreases with the increase in flow.

\section{Conflict of Interests}

The authors declare that there is no conflict of interests regarding the publication of this paper.

\section{Acknowledgment}

N. Kumar would like to acknowledge the support from IUCAA, Pune, India.

\section{References}

[1] C. E. DeForest and J. B. Gurman, "Observation of quasiperiodic compressive waves in solar polar plumes," Astrophysical Journal Letters, vol. 501, no. 2, pp. L217-L220, 1998.

[2] L. Ofman, M. Romoli, G. Poletto, G. Noci, and J. L. Kohl, "Ultraviolet Coronagraph Spectrometer observations of density fluctuations in the solar wind," Astrophysical Journal Letters, vol. 491, no. 2, pp. L111-L114, 1997.

[3] I. De Moortel, J. Ireland, A. W. Hood, and R. W. Walsh, "The detection of 3 \& 5 min period oscillations in coronal loops," Astronomy and Astrophysics, vol. 387, no. 1, pp. L13-L16, 2002.

[4] I. De Moortel, J. Ireland, R. W. Walsh, and A. W. Hood, "Longitudinal intensity oscillations in coronal loops observed with trace: I. Overview of measured parameters," Solar Physics, vol. 209, no. 1, pp. 61-88, 2002.

[5] V. M. Nakariakov, L. Ofman, E. E. Deluca, B. Roberts, and J. M. Davila, "Trace observation of damped coronal loop oscillations: implications for coronal heating," Science, vol. 285, no. 5429, pp. 862-864, 1999.

[6] M. Carbonell, R. Oliver, and J. L. Ballester, "Time damping of linear non-adiabatic magnetohydrodynamic waves in an unbounded plasma with solar coronal properties," Astronomy and Astrophysics, vol. 415, no. 2, pp. 739-750, 2004.

[7] M. Carbonell, J. Terradas, R. Oliver, and J. L. Ballester, "Spatial damping of linear non-adiabatic magnetoacoustic waves in a prominence medium," Astronomy and Astrophysics, vol. 460, no. 2, pp. 573-581, 2006.

[8] R. Soler, R. Oliver, and J. L. Ballester, "The effect of the solar corona on the attenuation of small-amplitude prominence oscillations I. Longitudinal magnetic field," Astronomy and Astrophysics, vol. 471, no. 3, pp. 1023-1033, 2007.

[9] A. H. Gabriel, F. Bely-Dubau, and P. Lemaire, “The contribution of polar plumes to the fast solar wind," Astrophysical Journal Letters, vol. 589, no. 1, pp. 623-634, 2003.
[10] J. M. Krijger, T. Roudier, and M. Rieutord, "Photospheric flows measured with TRACE," Astronomy and Astrophysics, vol. 387, no. 2, pp. 672-677, 2002.

[11] R. Soler, R. Oliver, and J. L. Ballester, "Nonadiabatic magnetohydrodynamic waves in a cylindrical prominence thread with mass flow," Astrophysical Journal Letters, vol. 684, no. 1, pp. 725735, 2008.

[12] V. M. Nakariakov and B. Roberts, "Magnetosonic waves in structured atmospheres with steady flows-I. Magnetic slabs," Solar Physics, vol. 159, no. 2, pp. 213-228, 1995.

[13] M. Carbonell, R. Oliver, and J. L. Ballester, "Time damping of non-adiabatic MHD slow and thermal waves in a prominence medium: effect of a background flow," New Astronomy, vol. 14, no. 3, pp. 277-284, 2009.

[14] S. Poedts, A. D. Rogava, and S. M. Mahajan, "Shear-flowinduced wave couplings in the solar wind," Astrophysical Journal Letters, vol. 505, no. 1, pp. 369-375, 1998.

[15] V. M. Nakariakov, C. A. Mendoza-Briceño, and M. H. Ibáñez S., "Magnetoacoustic waves of small amplitude in optically thin quasi-isentropic plasmas," Astrophysical Journal Letters, vol. 528, no. 2, pp. 767-775, 2000.

[16] T. V. Zaqarashvili, R. Oliver, and J. L. Ballester, in Proceedings of the SOHO 13, Waves Oscillations and Small-Scale Transient Events in the Solar Atmosphere: A Joint View from SOHO and TRACE, no. ESASP-547, Palma de Mallorca, Spain, 2004.

[17] B. P. Pandey and M. Wardle, "Hall instability of solar flux tubes in the presence of shear flow," Monthly Notices of the Royal Astronomical Society, vol. 426, pp. 1436-1443, 2012.

[18] B. P. Pandey and M. Wardle, "Magnetic-diffusion-driven shear instability of solar flux tubes," Monthly Notices of the Royal Astronomical Society, vol. 431, pp. 570-581, 2013.

[19] H. Saleem, J. Vranjes, and S. Poedts, "Unstable drift mode driven by shear plasma flow in solar spicules," Astronomy and Astrophysics, vol. 471, no. 1, pp. 289-293, 2007.

[20] G. D. Aburjania, G. Zimbardo, and O. A. Kharshiladze, "Effect of the shear flow in the generation and self-organization of internal gravity wave structures in the dissipative ionosphere," Plasma Physics Reports, vol. 38, no. 12, pp. 1055-1075, 2012.

[21] E. Hildner, "The formation of solar quiescent prominences by condensation," Solar Physics, vol. 35, no. 1, pp. 123-136, 1974.

[22] N. Kumar and P. Kumar, "Viscous damping of non-adiabatic MHD waves in an unbounded solar coronal plasma," Solar Physics, vol. 236, no. 1, pp. 137-153, 2006. 

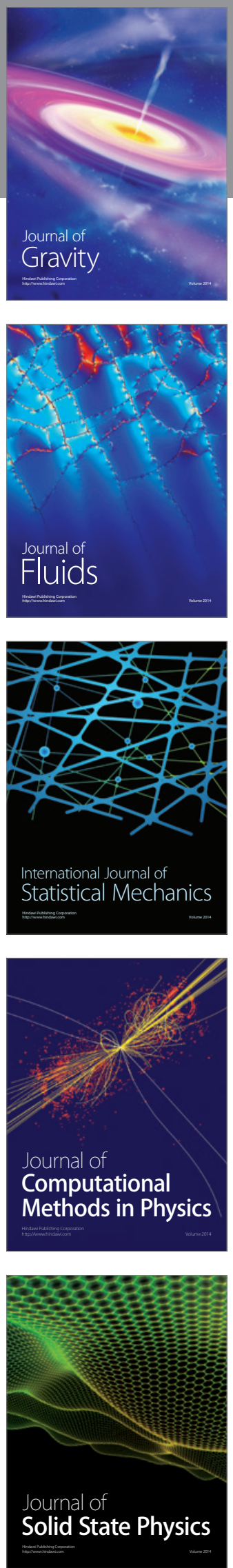

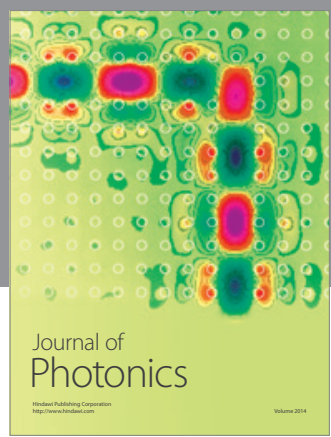

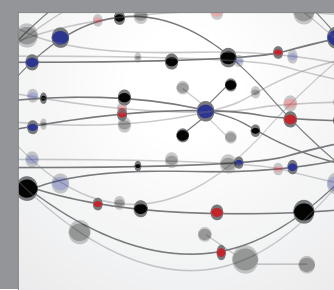

The Scientific World Journal

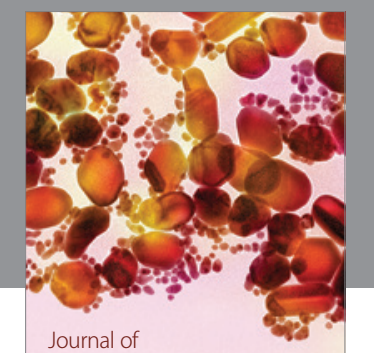

Soft Matter
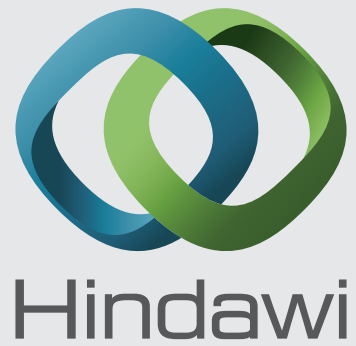

Submit your manuscripts at

http://www.hindawi.com
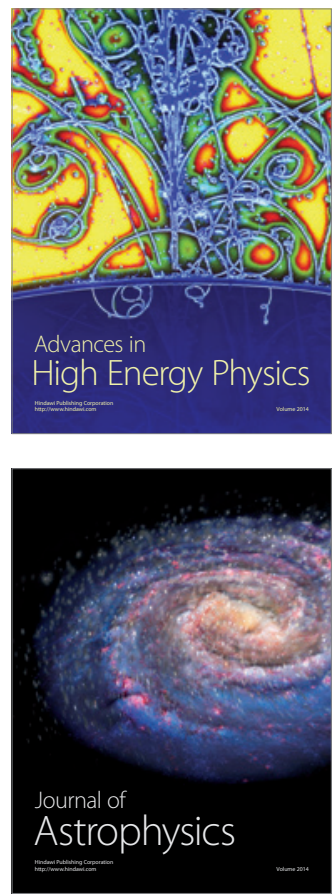
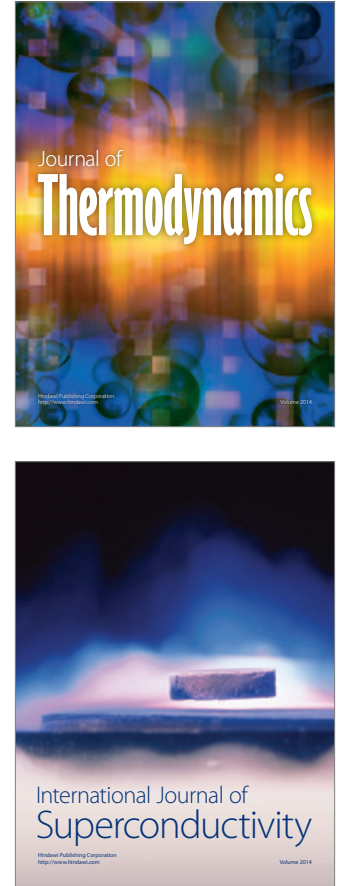
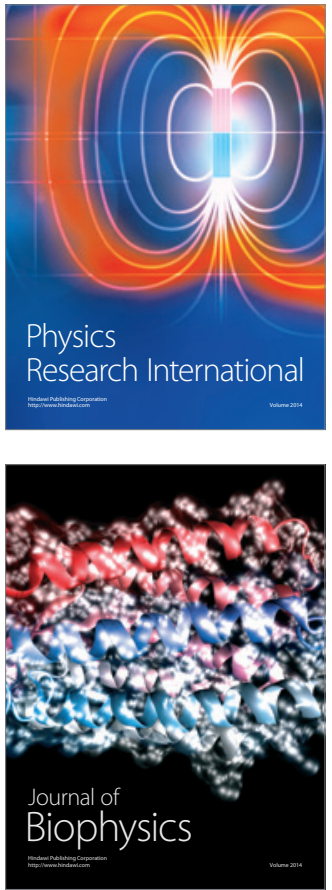
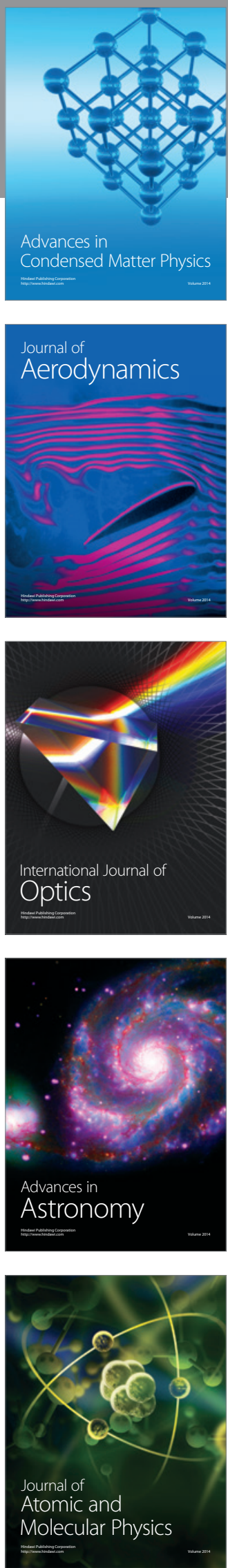